J. Biosoc. Sci., (2013) 45, 289-310, (C) Cambridge University Press, 2012. The online version of this article is published within an Open Access environment subject to the conditions of the Creative Commons Attribution-NonCommercial-ShareAlike licence <http://creativecommons.org/licenses/ by-nc-sa/3.0/>. The written permission of Cambridge University Press must be obtained for commercial re-use.

doi:10.1017/S0021932012000612 First published online 15 Nov 2012

\title{
TESTING EVOLUTIONARY THEORIES OF DISCRIMINATIVE GRANDPARENTAL INVESTMENT
}

\section{RALF KAPTIJN*, FLEUR THOMESE*, AART C. LIEFBROER*† AND MERRIL SILVERSTEIN:}

*VU University Amsterdam, the Netherlands, $\uparrow$ Netherlands Interdisciplinary Demographic Institute, The Hague, the Netherlands and $\vdots$ University of Southern California, Los Angeles, USA

\begin{abstract}
Summary. This study tests two evolutionary hypotheses on grandparental investments differentiated by the child's sex: the paternity uncertainty hypothesis and the Trivers-Willard hypothesis. Data are from two culturally different countries: the Dutch Longitudinal Aging Study Amsterdam $(n=2375)$ and the Chinese Anhui Survey $(n=4026)$. In the Netherlands, grandparental investments are biased towards daughters' children, which is in accordance with the paternity uncertainty hypothesis. But in China, grandparental investments are biased towards sons' children, which is in conflict with the paternity uncertainty hypothesis. This study found no support for the Trivers-Willard hypothesis. These results raise doubts over the relevance of paternity uncertainty as an explanation of a grandparental investment bias towards daughters' children that is often found in Western populations. The results suggest that discriminative grandparental investments are better understood as the outcome of cultural prescriptions and economic motives.
\end{abstract}

\section{Introduction}

In contemporary affluent societies, increases in life expectancy have enhanced the opportunities for grandparents to care for their grandchildren (Uhlenberg, 1996; Friedman et al., 2008; Coall \& Hertwig, 2010). These enhanced opportunities for grandparental childcare created by the larger shared lifespan between grandparents and grandchildren are frequently utilized. In the United States $23 \%$ of the children under 5 years of age are weekly cared for by their grandparents (Johnson, 2005) and $60 \%$ of grandparents provide occasional or more frequent care (Fuller-Thomson \& Minkler, 2001). A study in Europe found that $58 \%$ of grandmothers and $49 \%$ of grandfathers took care of at least one of their grandchildren in the preceding year (Hank \& Buber, 2009).

Grandparental childcare is potentially advantageous for both children and grandchildren. The quality of grandparental childcare is valued more highly than formal 
childcare by British as well as Dutch parents. Both British and Dutch parents mention the importance of trust for this preference (Wheelock \& Jones, 2002; Portegijs et al., 2006). Grandparents are thought to provide care that meets the specific needs of the grandchildren (Wheelock \& Jones, 2002). Grandparental childcare is also less expensive than formal childcare (Portegijs et al., 2006). Beside the perceived quality and low price of grandparental childcare this care could ease women's dilemma of combining paid employment and motherhood (Hoppmann \& Klumb, 2010). Grandparental childcare could allow the mother to maintain or increase her labour force participation (Cardia \& Ng, 2003; Gray, 2005; Dimova \& Wolff, 2011) and might make it easier for her to have more children (Kaptijn \& Thomese, 2010; Kaptijn et al., 2010). Although grandparental childcare can also be a source of conflict between grandparents and children, these conflicts are usually perceived as manageable (Wheelock \& Jones, 2002).

Grandparental investments in general are not divided equally among all children. A majority of older women have reported differentiating their investments among their adult children (Suitor et al., 2006, 2007). In explaining these differential investments, cultural, economic and evolutionary perspectives have all focused on different explanatory factors while rarely taking account of each other's views (Coall \& Hertwig, 2010). Cultural explanations have focused on values and norms and found that parents who hold an unconditional family solidarity norm invest more in their children (Kohli \& Künemund, 2003), that parents invest more in children who are more in need (McGarry \& Schoeni, 1997; Suitor et al., 2006, 2007; Fingerman et al., 2009) and that parental investments are lower for children who had problems with substance use or law enforcement (Suitor \& Pillemer, 2000). Economic explanations have focused on reciprocity and found that parents invest more in children who also provide support to them (Kunemund \& Rein, 1999; Boerner \& Reinhardt, 2003; Pillemer \& Suitor, 2006; Suitor et al., 2006) and that parents receive more support from children in whom they invested more heavily in the past (Henretta et al., 1997; Silverstein et al., 2002). Evolutionary explanations have focused on the adult child's sex and found that maternal grandparents invest more than paternal grandparents (Euler \& Weitzel, 1996; Michalski \& Shackelford, 2005; Pollet et al., 2009). However, evolutionary studies of inter-generational transfers in particular have been criticized for ignoring the importance of cultural factors for grandparental investments (Silverstein, 2007; Friedman et al., 2008; Gilding, 2009).

This study focuses on two key evolutionary hypotheses concerning grandparental investments differentiated by the child's sex while also taking account of cultural and economic explanations. Within the Darwinian paradigm, grandparental investments are understood as part of an evolved strategy in old age aimed at increasing the grandparents' reproductive success through the survival of their progeny. Especially women could gain fitness with these investments by increasing the reproductive success of their offspring rather than by continuing to reproduce themselves (Hawkes, 2004; Lahdenpera et al., 2004, 2007; Hrdy, 2005). This view, although debated (Strassmann \& Garrard, 2011), is supported by several studies in pre-modern populations, which found that the presence of a grandmother has a positive effect on the survival chances of the grandchild (reviewed by Sear \& Mace, 2008). In modern societies almost all children stay alive after birth and grandparents are not expected to contribute to the survival chances of their children. However, human behaviour in modern societies may still be explained by the vestiges of adaptations formed in pre-modern societies 
(Tooby \& Cosmides, 1990, 1992; Buss, 1994; Geary, 1998). In line with this reasoning, discriminative grandparental investments in modern societies have been explained by differences in the certainty of genetic relatedness between grandparents and grandchildren and differences in the reproductive potential of the children (e.g. Euler \& Weitzel, 1996; Chrastil et al., 2006; Pollet et al., 2006, 2009; Bishop et al., 2009). Grandparents are more certain of the genetic relationship with their daughters' children than with their sons' children, so grandparents are expected to invest more in their daughters' children than in their sons' children (Euler \& Weitzel, 1996). However, under good socioeconomic conditions sons are expected to produce more children than daughters (Hopcroft, 2006; Fieder \& Huber, 2007; Nettle \& Pollet, 2008). This would direct grandparental investments to the sons' children under good socioeconomic conditions (Trivers \& Willard, 1973), the potential reproductive benefit partly overcoming uncertainty over paternity. Below these two hypotheses are discussed in more detail.

\section{The paternity uncertainty hypothesis}

From an evolutionary perspective the main objective of parental investments is to enable one's own children to survive and reproduce. Although both men and women have an evolutionary interest in investing in their children, men and women are unequal in the certainty that their parental investments will actually benefit their biological children. Women are absolutely certain that the children they bear are their biological children. In contrast, men are less than $100 \%$ certain that they are the biological father of their putative children (Trivers, 1972). Even if men feel certain about their paternity, they run the risk of unknowingly investing in the children of somebody else. Based on a review of 67 studies, Anderson (2006) estimated the current rate of non-paternity for men with high paternity confidence to be $1.9 \%$. The risk of investing in somebody else's children is thought to shape men's parenting decisions. In line with this idea, men invest less in their children when the physical resemblance with their putative children is smaller (Alvergne et al., 2009; Heijkoop et al., 2009) and when they are less certain about their paternity (Anderson et al., 2007).

Because grandparents also run the risk of investing in genetically unrelated grandchildren when they invest in their sons' children, paternal grandparents are expected to invest less than maternal grandparents. Numerous studies have tested this hypothesis using a wide range of outcome measures and methods. Paternal grandparents are found to have less contact with their grandchildren (Salmon, 1999; Pollet et al., 2006, 2009), to be emotionally less close to their grandchildren (Laham et al., 2005; Michalski \& Shackelford, 2005; Bishop et al., 2009), to take less care of their grandchildren (Euler \& Weitzel, 1996; Euler et al., 2001; Chrastil et al., 2006) and to give less financial support to their grandchildren (Pollet et al., 2009). These results are obtained using self-reports from the grandchildren (Salmon, 1999; Laham et al., 2005; Bishop et al., 2009), the grandparents (Michalski \& Shackelford, 2005; Pollet et al., 2007, 2009) and retrospective reports of adult grandchildren (Euler \& Weitzel, 1996; Euler et al., 2001; Chrastil et al., 2006).

Although the results of these studies are consistent and could be interpreted as support for the paternity uncertainty hypothesis, these studies are limited in their use of samples from Western populations. The results are also in accordance with cultural 
explanations (Dubas, 2001; Friedman et al., 2008). Within Western cultures, women are said to be socialized as kin keepers. That is, women usually are more involved in family relationships and bear the prime responsibility for maintaining the family bonds. Thus, the larger investments of maternal grandparents compared with paternal grandparents might also be a consequence of women being socialized to have stronger bonds with their parents than men (Eisenberg, 1988; Dubas, 2001). Moreover, paternal grandparents do not always seem to invest less. Grandchildren in farm families in Iowa reported more frequent contact and higher quality relationships with their paternal grandparents (King \& Elder, 1995; King et al., 2003). Also in rural Greece, grandchildren reported having higher quality relationships with their paternal grandparents (Pashos, 2000). Although the authors themselves explained these results by reference to the rural patrilineal culture (King \& Elder, 1995; Pashos, 2000; King et al., 2003), these studies have been criticized for not truly measuring grandparental investments, but attempts of the middle generation to acquire land from the grandparents (Michalski \& Shackelford, 2005).

A second limitation of the above-mentioned studies is that they do not take reciprocity into account as an alternative explanation of the observed smaller investments of paternal grandparents. Exchange and delayed exchange might be important motives of the grandparents to invest in their grandchildren (Kohli \& Künemund, 2003; Friedman et al., 2008). In accordance with this idea parents invest more in adult children who also provide support to them (Kunemund \& Rein, 1999; Boerner \& Reinhardt, 2003; Pillemer \& Suitor, 2006; Suitor et al., 2006). The grandparental investment bias toward maternal offspring thus might be partly explained in terms of stimulating reciprocal support from daughters, particularly as future caregivers (Friedman et al., 2008).

In order to evaluate the relevance of the paternity uncertainty hypothesis beside cultural or economic explanations of biases towards daughters' children in grandparental investments, this hypothesis is tested in this study using data from two culturally different countries: the Netherlands and China. The Netherlands is a typical Western county where a grandparental investment bias towards daughters' children has been reported (Pollet et al., 2006, 2007). China has a patrilineal culture where sons are valued more than daughters, especially in rural areas (Lai-wan et al., 2006). To account for reciprocity, emotional and instrumental support that the grandparent received from the children is included in the analysis of both populations. To meet the critique on previous studies of not truly measuring grandparental investments, the provision of grandparental childcare is used as a direct measure of grandparental investment. This measure of grandparental investment has also been reported to be positively related to the children's fertility in the Netherlands (Kaptijn et al., 2010), which makes it a relevant indicator of grandparental investment from an evolutionary perspective.

\section{The Trivers-Willard hypothesis}

In addition to the certainty that grandparental investments actually benefit genetically related grandchildren, the reproductive potential of the children is also expected to play a role in discriminative grandparental investments. From an evolutionary point of view, grandparents should have an incentive to devote more of their resources to 
children who can be expected to produce the largest number of grandchildren. Because on the marriage market the value of occupying a high socioeconomic position differs between the sexes, the reproductive potential of sons and daughters also differ according to their socioeconomic position. In general, women tend to prefer men of high socioeconomic status, while men find the socioeconomic status of a potential partner less important (Buss \& Schmitt, 1993; Geary et al., 2004). As a result, high status men are more likely to marry than lower status men, leaving lower status men more often unmarried and childless, while for women such a pattern does not occur (Fieder \& Huber, 2007; Brown et al., 2009; Fieder et al., 2011). In most human populations, especially in serial monogamous and polygamous societies, variance in men's fertility exceeds variance in women's fertility (Brown et al., 2009). The effect of men's social status on their reproductive success is stronger in hunter-gatherer societies (Nettle \& Pollet, 2008), but also in contemporary serial monogamous societies, the relationship between socioeconomic status and fertility is positive for men, but negative for women (Hopcroft, 2006; Fieder \& Huber, 2007; Nettle \& Pollet, 2008; Fieder et al., 2011). For these reasons higher status sons are expected to produce more children than higher status daughters, whereas lower status daughters are expected to produce more children than lower status sons.

The hypothesis that parental investments - and by extension grandparental investments - are biased towards the child with the largest reproductive potential was originally formulated by Trivers \& Willard (1973) and became known as the TriversWillard hypothesis. Trivers \& Willard (1973) predicted a bias in the sex ratio of the children towards sons under good material conditions and towards daughters under poor material conditions. Tests of the Trivers-Willard hypothesis in contemporary modern societies have yielded mixed results. Parents in good conditions seem to have a larger chance of getting sons (Hopcroft, 2005; Almond \& Edlund, 2007; Magnuson et al., 2007; Cameron \& Dalerum, 2009), but parental investments are not always biased towards the sex with the largest reproductive potential (Gaulin \& Robbins, 1991; Freese \& Powell, 1999; Keller et al., 2001; Koziel \& Ulijaszek, 2001; Hopcroft, 2005).

Since, by taking care of the grandchildren, grandparents could facilitate their children's fertility (Kaptijn \& Thomese, 2010) and enhance the success of existing grandchildren (Coall \& Hertwig, 2010), grandparental investments are expected to be biased towards children of the sex with the largest reproductive potential, namely higher status sons and lower status daughters. The authors know of only one study that tests the Trivers-Willard hypothesis using grandparental investments. Euler \& Weitzel (1996) examined interaction effects between the parents' social status and sex on grandparents' solicitude with their grandchildren. They did not find support for the Trivers-Willard hypothesis in their study, but the sample of college students they used might have shown too little variance on socioeconomic conditions. In contrast with Euler \& Weitzel (1996), this study uses representative samples of the older population of the Netherlands and rural China. The use of culturally different populations allows acknowledgment of possible cultural factors in how investment strategies used by grandparents vary by the sex and socioeconomic status of the children. Further, emotional and instrumental support received from the adult children of grandparents is included to account for reciprocity as a motivation for grandparental investments. 
To recapitulate, two evolutionary mechanisms are relied upon to explain discriminative grandparental investments. The paternity uncertainty hypothesis predicts that grandparents bias their investments towards their daughters' children, because grandparents are more certain of their genetic relationship with their daughters' children than with their sons' children. The Trivers-Willard hypothesis predicts that grandparents bias their investments more towards their sons' children under good socioeconomic conditions and more towards their daughters' children under poor socioeconomic conditions because, due to marital selection processes based on socioeconomic position that are highly gendered, high status sons are expected to produce more grandchildren than high status daughters and the other way around for children with a lower socioeconomic status. These two mechanisms may operate simultaneously such that a general bias in grandparental investments towards maternal grandchildren may be reduced or shift to a bias towards paternal grandchildren under good socioeconomic conditions, but may be enlarged under adverse socioeconomic conditions.

Although grandparental investments in modern society might convey a fitness benefit for the grandparents, and Dutch parents that received childcare support from the grandparents had a higher probability of having additional children (Kaptijn et al., 2010; but see Waynforth, 2012), this hypothesis is not tested in this paper. Paternity uncertainty and a Trivers-Willard effect are thought to have shaped grandparental investment decisions during the human evolutionary past (Euler \& Weitzel, 1996; Pollet et al., 2006; Bishop et al., 2009). It is assumed that grandparents in modern societies may still invest according to these earlier evolutionary pressures even if this behaviour is no longer maximizing grandparental fitness in modern societies (Tooby \& Cosmides, 1990, 1992; Buss, 1994; Geary, 1998). This study tests if discriminative grandparental investments in modern societies are in line with the paternity uncertainty hypothesis and the Trivers-Willard hypothesis, irrespective of the reproductive consequences of these investments.

The study also analyses grandfathers' investments in their grandchildren. In contrast to grandmothers, who experience menopause, grandfathers could gain fitness by continuing to reproduce in old age instead of investing in their grandchildren. Opportunities for men to reproduce at old age were widespread in pre-modern polygamous societies, but in modern serial monogamous societies these opportunities are much more limited (Tuljapurkar et al., 2007). There is not much evidence that grandfathers were beneficial for grandchildren's survival in pre-modern societies in the same way as grandmothers were (Sear \& Mace, 2008). Still, in evolutionary studies on discriminative grandparental investments, grandfathers' investments in their grandchildren in modern societies are commonly understood as an evolved reproductive strategy of men at old age (e.g. Euler \& Weitzel, 1996; Michalski \& Shackelford, 2005; Pollet et al., 2006, 2007; Bishop et al., 2009). In this study grandfathers' investments in their grandchildren are included to examine if evolutionary explanations also hold for grandfathers' investments.

\section{Samples}

\section{Methods}

The Netherlands. The sample of the Dutch older population is derived from the survey on Living arrangements and Social Networks of older adults (LSN; Knipscheer 
et al., 1995) and its follow-up, the Longitudinal Aging Study Amsterdam (Deeg et al., 2002). The LSN survey is a representative sample of the Dutch older population stratified by age and sex. Face-to-face interviews were conducted in 1992 when respondents were aged 55 to 89. In 1992, a random sample of 729 grandparents in the LSN survey were asked about the childcare they gave to each individual grandchild aged 16 or younger. In 2002, a new cohort of people aged 55 to 64 was included in the sample of the Longitudinal Aging Study Amsterdam (LASA). From this new cohort, a random sample of 544 grandparents were asked about the childcare they gave to their grandchildren. The units of analysis are the children of the respondents. Only the children who were parents themselves were included in the sample. Information on the children was collected through interviews with the grandparent. The 1273 grandparents that were interviewed about their grandchildren had 2499 children in total. Because the paternity certainty hypothesis and Trivers-Willard hypothesis only refer to presumably biological children, all stepchildren and adopted children were excluded from the sample, leaving 2407 biological children eligible for analysis. Due to missing values on the relevant variables the final sample consisted of 2375 children.

China. The sample of the Chinese older population comes from the Anhui survey of 2001. Anhui, a mostly rural province of China, is characterized by a relative high proportion of older adults and high levels of out-migration of working-age adults. The sample was drawn using a multistage method. In the first stage, twelve rural townships were randomly selected from the Chaohu region, located in the central part of Anhui. In the second stage, six administrative villages were randomly selected from each township. In the third stage, a random selection was drawn of the inhabitants of these villages aged 60 and over with an over-sampling of people aged 75 and over. The initial sample included 1421 grandparents who were asked about the childcare they provided to their grandchildren aged 16 or younger. These grandparents had 4289 children in total. The Anhui survey did not include questions about stepchildren or adopted children, so these could not be excluded. For 263 children there was information missing on one or more of the relevant variables, leaving a final sample of 4026 children. These missing cases are for $49 \%$, due to grandparents not knowing the age of their children or grandchildren.

\section{Measures}

Dependent variable. The dependent variable is grandparental childcare provisioning. In the Dutch sample, the grandparents were asked how much they had taken care of each individual grandchild aged 16 or younger in the past 12 months. Possible answer categories were: 'never', 'seldom', 'sometimes' and 'often'. Although this question was answered for each individual grandchild, there was not much variance at the grandchild level. In most (93\%) cases, the grandparent took care of all grandchildren of the same child equally often. Therefore, it was decided to aggregate this variable to the child level by taking the maximum frequency of grandparental childcare.

In the Chinese sample the grandparents were asked how much they had taken care of any of the grandchildren from a specific child in the preceding 12 months. Possible answer categories were: 'never', 'less than once a month', 'every month or so', 'several 
times per month', 'at least once per week', 'daily, or more often, but not all day' and 'full-day custody'.

Independent variables. In both the Dutch and the Chinese sample, the sex of the children is derived from the interview with the grandparent. The Trivers-Willard hypothesis was tested with an interaction term between child's sex and grandparental socioeconomic position. Information on the child's socioeconomic condition was not available in the Dutch sample, so grandparental socioeconomic condition was used as a proxy for the child's socioeconomic position in both samples. A separate analysis was run using an interaction term between child's socioeconomic position (i.e. educational achievement) and child's sex in the Chinese data. The conclusions from this separate analysis were the same as the ones presented here. The measure of grandparental socioeconomic condition is grandparental educational achievement. In the Dutch sample, educational achievement was measured on a nine-point ordinal scale. In the Chinese sample, educational achievement was measured on a seven-point ordinal scale. In order to enhance comparability across both populations this measure was dichotomized by a median split. In the Dutch sample, grandparents with a lower vocational education or higher belong to the more educated group. In the Chinese sample, grandparents with primary education or higher belong to the more educated group. One could argue that educational achievement does not differentiate well on grandparental socioeconomic condition in China, because $77 \%$ of the elderly in the sample did not get any education at all. In order to address this issue, the Trivers-Willard hypothesis was additionally tested in China using grandparental income and house ownership as alternative measures of socioeconomic condition. These additional analyses led to the same conclusions as the ones presented in this paper.

Control variables. A number of possible confounding factors at the grandparent level and the child level were controlled for. In most cases, the measurement of these variables does not differ between the Dutch and the Chinese samples. In this paper, the cases in which the measurement in both populations is different will be explicitly referred to. At the grandparent level, grandparent's sex, having a partner living in the same household, the grandparent's educational achievement, the grandparent's health and the number of children of the grandparent who are parents of children aged 16 or younger are controlled for. Educational achievement is measured as described above. Health is measured as self-rated health. In the Dutch sample, there were five answer categories. These were 'poor', 'not so good', 'fair', 'good' and 'very good'. In the Chinese sample, there were four answer categories: 'poor', 'fair', 'good' and 'excellent'. In order to enhance comparability, both were standardized. Because the Dutch sample consisted of two cohorts the cohort in the Dutch sample was additionally controlled for.

At the child level, having a partner living in the same household, the geographical distance between the child and the grandparent, the child's age, the age of the grandparent relative to the child's age, the age of the youngest grandchild relative to the child's age, the number of grandchildren and the emotional and instrumental support that the grandparent received from the child were controlled for. In the Dutch sample, the distance between the child and the grandparent was measured as the travel time for the grandparent to the child (in minutes, log transformed). In the Chinese sample, the 
distance between the child and the grandparent was measured on an ordinal scale. Grandparents were asked the question where the child lived. The answer categories were: 'in the same household', 'in the same village', 'in the same township', 'in the same county', 'in the same city', 'in the same province', 'in China' and 'outside China'. Because only four children lived abroad the categories 'in China' and 'outside China' were merged into the category 'outside the province'. The child's age, the grandparent's age and the age of the youngest grandchild were strongly correlated. In order to be able to include these three variables in the model, the standardized residual of the grandparent's age and the standardized residual of the age of the youngest grandchild were used, both after regression on the age of the child. A positive value on these variables means that the grandparent or youngest grandchild is relatively old given the child's age.

In the Dutch sample, the questions regarding emotional and instrumental support were only asked if the child was identified as a member of the personal network of the grandparent: that is, if the grandparent had regular contact with the child and the grandparent considered this contact important. In addition, these questions were only asked if the child belonged to one of the twelve (or ten, in case of the younger cohort) network members with whom the grandparent had the most frequent contact. Because of this procedure, the scores on emotional and instrumental support were not available for $24 \%$ of the children. A dummy was constructed indicating if the scores on emotional and instrumental support were missing and the mean for the children whose scores on these variables were not available was imputed. A separate analysis was run in which all cases with missing values on emotional or instrumental support were excluded. The conclusions from this separate analysis were the same as the ones presented in this paper. Emotional support is measured with the question 'How often did it occur in the last year that you told [your child] about your personal experiences and feelings?' and instrumental support is measured as 'How often did it occur in the last year that [your child] helped you with daily chores in and around the house?' Both variables were measured on a four-point scale ranging from 'never' to 'often'. In the Chinese sample emotional support is measured with the question: 'How much do you feel that [your child] would be willing to listen when you need to talk about your worries and problems?' Possible answer categories were: 'not at all well', 'somewhat well' and 'quite well'. Instrumental support was operationalized as the value, in Chinese Yuan, of the money, food or gifts that the child had given to the grandparent in the preceding year. Instrumental support was measured on a ten-point scale. All measures of emotional and instrumental support were standardized to improve the comparability of these measures across both populations. Descriptive statistics of all variables are shown in Table 1 for the Dutch sample and Table 2 for the Chinese sample.

\section{Statistical analysis}

The data have a hierarchical structure. The children are nested within the grandparents. The dependent variables are ordinal. An ordinal multilevel regression analysis was conducted to test the hypotheses using the Markov Chain Monte Carlo (MCMC) method to estimate the parameters of the model. This is the preferred estimation 
R. Kaptijn et al.

Table 1. Descriptive statistics of the Dutch sample

\begin{tabular}{llll}
\hline & Mean $^{\mathrm{a}}$ & $\mathrm{SD}^{\mathrm{b}}$ & Range $^{\mathrm{b}}$ \\
\hline Grandparent level & & & \\
Grandmother & $55 \%$ & & \\
Partner in household & $75 \%$ & & \\
Educated & $64 \%$ & 1.12 & $1-5$ \\
Health & 3.11 & 1.19 & $1-10$ \\
Number of children & 1.98 & & \\
Young cohort & $42 \%$ & 7.74 & \\
Age (years) & 67.65 & & \\
No. grandparents & 1240 & & \\
Child level & & & \\
Daughter & $52 \%$ & $1.00-7.27$ \\
Partner in household & $95 \%$ & 6.12 & $0-16$ \\
Travel time (minutes, log) & 3.10 & 4.75 & $1-8$ \\
Age (years) & 36.68 & 0.86 & $1-4$ \\
Age youngest grandchild (years) & 6.08 & 0.86 & \\
Number of grandchildren & 1.93 & 0.94 & \\
Emotional support to grandparent & 2.96 & & \\
Instrumental support to grandparent & 2.11 & & \\
Support to grandparent not available & $24 \%$ & & \\
Grandparental childcare provisioning & & & \\
Never & $44 \%$ & & \\
Seldom & $8 \%$ & & \\
Sometimes & $25 \%$ & & \\
Often & $23 \%$ & & \\
No. children & 2375 & & \\
\hline Pentage & & & \\
\hline
\end{tabular}

${ }^{\text {a }}$ Percentage shown if variable is dichotomous.

${ }^{\mathrm{b}}$ Not shown if variable is dichotomous.

method for ordinal multilevel models because it generates reliable interval estimates of the parameters of non-linear multilevel models (Rasbash et al., 2004; Draper, 2008). The models have two levels, with children at level one and grandparents at level two. The models' intercepts are random and have a common variance on the grandparent level. All independent and control variables were entered to the model as fixed effects.

Probit models were estimated to compare the regression coefficients between both populations. The probit model assumes an underlying latent continuous variable of grandparental childcare provisioning. The regression coefficients of ordinal probit models can be interpreted as the effect on the $Z$-score of that latent variable (Liao, 1994). For example, a regression coefficient of 0.5 means that a one-point increase in the independent variable leads to an expected increase of half a standard deviation in the latent continuous variable.

Because a grandparental investment bias towards daughters' children might be partly mediated by the emotional and instrumental support that the child gave to the grandparent, a stepwise analysis is performed. The paternity certainty hypothesis is tested in the first two models. Model 1 includes the child's sex and the control variables 
Table 2. Descriptive statistics of the Chinese sample

\begin{tabular}{|c|c|c|c|}
\hline & Mean $^{\mathrm{a}}$ & $\mathrm{SD}^{\mathrm{b}}$ & Range $^{\mathrm{b}}$ \\
\hline \multicolumn{4}{|l|}{ Grandparent level } \\
\hline Grandmother & $49 \%$ & & \\
\hline Partner in household & $60 \%$ & & \\
\hline Educated & $23 \%$ & & \\
\hline Health & 2.09 & 0.86 & $1-4$ \\
\hline Number of children & 3.06 & 1.42 & $1-8$ \\
\hline Age (years) & 69.61 & 6.54 & $58-89$ \\
\hline No. grandparents & 1361 & & \\
\hline \multicolumn{4}{|l|}{ Child level } \\
\hline Daughter & $46 \%$ & & \\
\hline Partner in household & $97 \%$ & & \\
\hline \multicolumn{4}{|l|}{ Distance from grandparent } \\
\hline Same household & $6 \%$ & & \\
\hline Same village (Ref.) & $28 \%$ & & \\
\hline Same township & $15 \%$ & & \\
\hline Same county & $14 \%$ & & \\
\hline Same city & $1 \%$ & & \\
\hline Same province & $7 \%$ & & \\
\hline Outside the province & $30 \%$ & & \\
\hline Age (years) & 36.21 & 5.61 & $21-61$ \\
\hline Age youngest grandchild (years) & 8.71 & 4.02 & $0-16$ \\
\hline Number of grandchildren & 1.78 & 0.82 & $1-12$ \\
\hline Emotional support to grandparent & 2.52 & 0.62 & $1-3$ \\
\hline Instrumental support to grandparent & 3.77 & 1.63 & $1-10$ \\
\hline \multicolumn{4}{|l|}{ Grandparental childcare provisioning } \\
\hline Never & $65 \%$ & & \\
\hline Less than once a month & $8 \%$ & & \\
\hline Every month or so & $1 \%$ & & \\
\hline Several times per month & $3 \%$ & & \\
\hline At least once per week & $4 \%$ & & \\
\hline Daily, or more often, but not all day & $12 \%$ & & \\
\hline Full-day custody & $7 \%$ & & \\
\hline No. children & 4026 & & \\
\hline
\end{tabular}

${ }^{a}$ Percentage shown if variable is dichotomous.

${ }^{\mathrm{b}}$ Not shown if variable is dichotomous.

except for emotional and instrumental support. Model 2 includes the child's sex and all control variables including emotional and instrumental support. The Trivers-Willard hypothesis is tested in a third model. This model includes the same variables as Model 2 plus the interaction term between child's sex and grandparental educational attainment. All independent and control variables were centred at their means so that the intercepts can be interpreted as the thresholds between the categories of the dependent variable (Liao, 1994). 


\section{Results}

The results of the analysis of the Dutch data are shown in Table 3. In the Dutch sample daughters receive significantly more grandparental childcare support than sons. This effect hardly changes with the addition of emotional and instrumental support in Model 2 , which shows that this effect is not mediated by reciprocity. The results of the Dutch data thus seem to be in accordance with the paternity uncertainty hypothesis. However, the analysis of the Chinese data in Table 4 shows that in China daughters receive significantly less grandparental childcare support than sons. Also in the Chinese data this effect is not mediated by instrumental or emotional support. The results of the Chinese data contradict the paternity uncertainty hypothesis.

The Trivers-Willard hypothesis is tested in Model 3 of Table 3 and Model 3 of Table 4. No evidence for the Trivers-Willard hypothesis was found in the analyses of

Table 3. Regression estimates for ordered probit multilevel models explaining the intensity of grandparental childcare provisioning in the Netherlands

\begin{tabular}{|c|c|c|c|}
\hline & Model 1 & Model 2 & Model 3 \\
\hline \multicolumn{4}{|l|}{ Grandparent level } \\
\hline Grandmother & $0.328^{* * *}$ & $0.294 * * *$ & $0.296^{* * *}$ \\
\hline Partner in household & $0.234^{* *}$ & $0.247 * *$ & $0.244^{* *}$ \\
\hline Educated & 0.077 & 0.060 & 0.106 \\
\hline Health (standardized) & $0.106^{*}$ & $0.097 *$ & $0.096^{*}$ \\
\hline Number of children & $-0.196 * * *$ & $-0.168 * * *$ & $-0.169 * * *$ \\
\hline Young cohort & $0.515^{* * *}$ & $0.476^{* * *}$ & $0.472 * * *$ \\
\hline \multicolumn{4}{|l|}{ Child level } \\
\hline Daughter & $0.397 * * *$ & $0.327 * * *$ & $0.380^{* * *}$ \\
\hline Partner in household & $-0.316^{*}$ & $-0.293^{*}$ & $-0.295^{*}$ \\
\hline Travel time (minutes, log) & $-0.393 * * *$ & $-0.364 * * *$ & $-0.365 * * *$ \\
\hline Age (years) & $-0.098 * * *$ & $-0.095 * * *$ & $-0.095 * * *$ \\
\hline Residual age grandparent & $-0.235^{* * *}$ & $-0.227 * * *$ & $-0.227 * * *$ \\
\hline Residual age youngest grandchild & $-0.341 * * *$ & $-0.328 * * *$ & $-0.329 * * *$ \\
\hline Number of grandchildren & 0.048 & 0.046 & 0.047 \\
\hline Emotional support to grandparent (standardized) & & $0.108 * *$ & $0.106 * *$ \\
\hline Instrumental support to grandparent (standardized) & & 0.017 & 0.018 \\
\hline Support to grandparent not available & & $-0.451 * * *$ & $-0.450 * * *$ \\
\hline Grandparent's education $\times$ daughter & & & -0.083 \\
\hline \multicolumn{4}{|l|}{ Thresholds } \\
\hline$\mu_{1}$ & $-1.242 * * *$ & $-1.252 * * *$ & $-1.255^{* * *}$ \\
\hline$\mu_{2}$ & $-0.140 * * *$ & $-0.138 * * *$ & $-0.140 * * *$ \\
\hline$\mu_{3}$ & $0.214 * * *$ & $0.220 * * *$ & $0.217 * * *$ \\
\hline Variance of intercepts ${ }^{\mathrm{a}}$ & 0.526 & 0.505 & 0.511 \\
\hline No. grandparents & 1240 & 1240 & 1240 \\
\hline No. children & 2375 & 2375 & 2375 \\
\hline
\end{tabular}

${ }^{a}$ Asterisks are not shown because MCMC confidence intervals of variance parameters always exclude 0 (Hox, 2002).

${ }^{*} p<0.05 ; * p<0.01 ; * * * p 0.001$. 
Table 4. Regression estimates for ordered probit multilevel models explaining the intensity of grandparental childcare provisioning in China

\begin{tabular}{|c|c|c|c|}
\hline & Model 1 & Model 2 & Model 3 \\
\hline \multicolumn{4}{|l|}{ Grandparent level } \\
\hline Grandmother & 0.058 & 0.046 & 0.048 \\
\hline Partner in household & $0.189^{* *}$ & $0.191 * *$ & $0.193^{* *}$ \\
\hline Educated & 0.092 & 0.081 & 0.107 \\
\hline Health (standardized) & $0.065^{*}$ & 0.061 & 0.062 \\
\hline Number of children & $-0.068 * *$ & $-0.070 * *$ & $-0.069 * *$ \\
\hline \multicolumn{4}{|l|}{ Child level } \\
\hline Daughter & $-0.926 * * *$ & $-0.931 * * *$ & $-0.916^{* * *}$ \\
\hline Partner in household & $-0.494 * * *$ & $-0.537 * * *$ & $-0.534 * * *$ \\
\hline \multicolumn{4}{|l|}{ Distance from grandparent } \\
\hline Same household & $1.207 * * *$ & $1.183^{* * *}$ & $1.186^{* * *}$ \\
\hline Same township & $-0.463 * * *$ & $-0.482 * * *$ & $-0.481^{* * *}$ \\
\hline Same county & $-0.395 * * *$ & $-0.429 * * *$ & $-0.431 * * *$ \\
\hline Same city & -0.381 & $-0.428^{*}$ & $-0.425^{*}$ \\
\hline Same province & -0.199 & $-0.241^{*}$ & $-0.243^{*}$ \\
\hline Outside the province & $0.267 * * *$ & $0.233 * * *$ & $0.231 * * *$ \\
\hline Age & $-0.060 * * *$ & $-0.060 * * *$ & $-0.060 * * *$ \\
\hline Residual age grandparent & $-0.141^{* * *}$ & $-0.143 * * *$ & $-0.143^{* * *}$ \\
\hline Residual age youngest grandchild & $-0.070 * *$ & $-0.071 * *$ & $-0.071 * *$ \\
\hline Number of grandchildren & $0.088 *$ & $0.092 *$ & $0.093^{*}$ \\
\hline Emotional support to grandparent (standardized) & & 0.017 & 0.018 \\
\hline Instrumental support to grandparent (standardized) & & $0.078 * *$ & $0.078 * *$ \\
\hline Grandparent's education $\times$ daughter & & & -0.071 \\
\hline \multicolumn{4}{|l|}{ Thresholds } \\
\hline$\mu_{1}$ & $-2.194 * * *$ & $-2.195 * * *$ & $-2.196^{* * *}$ \\
\hline$\mu_{2}$ & $-1.315 * * *$ & $-1.316^{* * *}$ & $-1.317 * * *$ \\
\hline$\mu_{3}$ & $-1.127 * * *$ & $-1.127 * * *$ & $-1.128 * * *$ \\
\hline$\mu_{4}$ & $-0.988 * * *$ & $-0.988 * * *$ & $-0.988 * * *$ \\
\hline$\mu_{5}$ & $-0.930 * * *$ & $-0.930 * * *$ & $-0.930^{* * *}$ \\
\hline$\mu_{6}$ & $-0.570 * * *$ & $-0.570 * * *$ & $-0.570^{* * *}$ \\
\hline Variance of intercepts ${ }^{\mathrm{a}}$ & 0.629 & 0.620 & 0.619 \\
\hline No. grandparents & 1361 & 1361 & 1361 \\
\hline No. children & 4026 & 4026 & 4026 \\
\hline
\end{tabular}

${ }^{\mathrm{a}}$ Asterisks are not shown because MCMC confidence intervals of variance parameters always exclude 0 (Hox, 2002).

$* p<0.05 ; * * p<0.01 ; * * *<0.001$.

the Dutch and the Chinese data. In both analyses the interaction term of grandparental educational achievement with child's sex is not statistically significant. In the Netherlands, more educated grandparents bias their childcare provisioning towards daughters' children as much as less educated grandparents do. In China, more educated grandparents bias their childcare provisioning towards sons' children as much as less educated grandparents do. 
The control variables in general show the importance of grandparents' opportunities and children's needs. Grandparents provide more care to their grandchildren when they have a partner living in the same household, when they are healthier, when they can divide their efforts over a smaller number of children and when they are younger relative to the child's age. Children receive more childcare when they do not have a partner living in the same household, when they are younger and when they have younger children. In the case of China children with a larger family also receive more childcare. In China, children living in the same household as the grandparent receive more grandparental childcare than children living in the same village as the grandparent. Exclusion of the children living in the same household as the grandparent did not change the conclusions. Children who live further away receive less grandparental childcare than children who live in the same village as the grandparent, though these effects are not statistically significant in all models. Children who live outside the province receive more grandparental childcare than children who live in the same village as the grandparent. Additional analyses revealed that this last effect is primarily due to grandparents providing more daily care and full-day custody to the grandchildren of these children living outside the province. The Dutch data did not show such a u-shaped relationship between travel time and the frequency of grandparental childcare provisioning.

The control variables further show that reciprocity also plays a role in explaining grandparental childcare provisioning, though there are also country-specific differences in this effect. In the Netherlands, children who provide more emotional support to the grandparent receive more grandparental childcare. Instrumental support has no significant effect in the Netherlands. The children whose scores on emotional and instrumental support to the grandparent were not available, because they were not identified as a member of the personal network of the grandparent, or because they did not have frequent contact with the grandparent, received less grandparental childcare. In China, emotional support has no statistically significant effect, but children who provide more instrumental support to the grandparent receive more grandparental support.

Finally, grandmothers provide more childcare than grandfathers in the Netherlands. In China this effect was not statistically significant. In the Netherlands, the younger cohort provides more care than the older cohort. The Chinese sample consisted of only one cohort. Grandparental educational achievement does not play a role in childcare provisioning in either population.

\section{Discussion}

Cultural, economic and evolutionary perspectives have all focused on different factors to explain discriminative grandparental investments, while rarely taking account of each other's views (Coall \& Hertwig, 2010). This study focused on two evolutionary hypotheses concerning grandparental investments differentiated by the child's sex while also taking account of cultural and economic explanations. The evolutionary hypotheses were tested in a contemporary Dutch and a contemporary rural Chinese population, two culturally and economically diverse societies, to account for cultural factors, and emotional and instrumental support was controlled for to account for reciprocity. 
According to the paternity uncertainty hypothesis, grandparents tilt their investments towards their daughters' children because they are more certain of their genetic relationship with their daughters' children than with their sons' children. In the Dutch sample a grandparental investment bias towards daughters' children was found, which is in accordance with this hypothesis, but is also consistent with the Dutch cultural orientation that favours mother-daughter bonds. In China, which predominantly has a patrilineal culture, a grandparental investment bias towards sons' children was found, which goes against the paternity uncertainty hypothesis. These results raise doubts over the relevance of paternity uncertainty as an explanation of the grandparental investment bias towards daughters' children that is often found in Western populations (e.g. Euler \& Weitzel, 1996; Michalski \& Shackelford, 2005; Chrastil et al., 2006; Bishop et al., 2009; Pollet et al., 2009).

Despite the risk of investing in genetically unrelated grandchildren, Chinese grandparents invest more in the children of their sons. One could argue that maybe paternity uncertainty is higher in the Netherlands than in China. To the authors' knowledge, there are no studies that compare the Dutch and Chinese paternity uncertainty rates. However, also, when paternity uncertainty in China is low, Chinese grandparents would still run the risk of investing in unrelated grandchildren. Even with low levels of paternity uncertainty the grandparental investment bias towards sons' children in China would be inconsistent with the paternity uncertainty hypothesis.

Although no consistent bias was found in grandparental investments towards daughters' children, these results do not imply that paternity uncertainty is irrelevant for grandparental investments in general. While at the societal level cultural prescriptions may be a dominant factor in investment decisions, at the family level they may reflect individual assessments of parental certainty (Silverstein, 2007) in the same way as men have been found to invest less in their putative children if they are less certain about their paternity (Anderson et al., 2007; Alvergne et al., 2009; Heijkoop et al., 2009). The behaviour or reputation of the daughter-in-law and the physical resemblance between the grandchildren and the grandparents' son might give cues to the certainty of the genetic relationship between grandparents and specific grandchildren. Grandparents might adjust their investments according to these cues. Testing the paternity uncertainty hypothesis in this way was beyond the scope of this study, but might be a fruitful path for future research.

According to the Trivers-Willard hypothesis, grandparents bias their investments more towards sons' children under good socioeconomic conditions and more towards daughters' children under adverse socioeconomic conditions. Our study found no evidence for this hypothesis in its analyses of the Dutch and Chinese data. The investment bias towards daughters' children in the Netherlands and sons' children in China was equal for more educated and less educated grandparents in both populations.

The test of the Trivers-Willard hypothesis is limited because grandparental socioeconomic condition was used as a proxy of the child's socioeconomic condition. However, in the Chinese sample the Trivers-Willard hypothesis was also tested using the child's educational achievement as a measure of socioeconomic condition. This test did not provide evidence for the Trivers-Willard hypothesis either. Chinese grandparents bias their investments towards sons' children, irrespective of the educational achievement of these sons. 
It may be that a Trivers-Willard effect at the grandparent level manifests itself only as a relationship between grandparental socioeconomic condition and sex ratio of the grandchildren, not as a relationship between grandparental socioeconomic condition and biased grandparental investments after the grandchildren are born. This would be in line with tests of the Trivers-Willard hypothesis at the parental level. Several studies on the relationship between parental socioeconomic condition and sex ratio have found a Trivers-Willard effect (Hopcroft, 2005; Magnuson et al., 2007; Almond \& Edlund, 2007; Cameron \& Dalerum, 2009), whereas studies that used measures of parental investment to test the Trivers-Willard hypothesis found much less support for this hypothesis (Freese \& Powell, 1999; Keller et al., 2001; Koziel \& Ulijaszek, 2001; Hopcroft, 2005). Further research may examine the relationship between grandparental socioeconomic condition or grandparental investments and the sex-ratio of the grandchildren for a Trivers-Willard effect.

Although this study was not designed as a test of cultural and reciprocal explanations of grandparental investments, the results do suggest that cultural conditions and reciprocity are relevant explanatory factors in discriminative grandparental investment. The observed grandparental investment bias towards daughters' children in the Netherlands is in line with the kin keeper hypothesis, and the grandparental investment bias towards sons' children in China could be explained by the patrilineal Chinese culture. The relevance of reciprocity is shown by the positive effect of the child's emotional support on grandparental investment in the Netherlands and the positive effect of the child's instrumental support on grandparental investment in China. The importance of emotional support in the Netherlands is possibly the result of the greater wealth of the Dutch grandparents relative to the Chinese grandparents. When grandparents are well-off materially, emotional support may become more important to them than instrumental support. Although the different operationalization of instrumental support instrumental support in the form of services in the Netherlands and instrumental support in the form of money and goods in China - limits the comparability of the effect of instrumental support in both populations, it is unlikely that this limited comparability has affected this study's conclusions. It was very uncommon for grandparents in the Netherlands to receive payment from their children for looking after the grandchildren at the time of data collection (Portegijs et al., 2006).

The aim of this study was to test two evolutionary hypotheses: the paternity uncertainty hypothesis and the Trivers-Willard hypothesis. However, the results suggest that discriminative grandparental investments are better understood as the outcome of cultural prescriptions and reciprocity than by these two evolutionary hypotheses. That is not to say that evolutionary processes in general are irrelevant for understanding discriminative grandparental investments. Human reciprocity and cultural changes can also be understood from an evolutionary point of view (Trivers, 1971; Richerson \& Boyd, 2004; Nowak, 2006). Human reciprocity is not at odds with evolution when both exchanging parties benefit from the exchange (Trivers, 1971; Nowak, 2006). In addition, human culture itself can be understood as an adaptation (Richerson \& Boyd, 2004; Boyd \& Richerson, 2009). Human survival and reproduction greatly benefited from social learning (Boyd et al., 2011). At the same time, it is important to realize that not all ideas that are spread by social learning are necessarily adaptive. The benefits of social learning come with the downside that people have to be credulous 
and conformist, leaving the possibility for maladaptive ideas to spread (see Richerson \& Boyd, 2004; Boyd \& Richerson, 2009). However, these alternative evolutionary explanations are commonly not referred to in the evolutionary literature concerning discriminative grandparental investments and were not tested in this study.

Further research might investigate the role of cultural prescriptions and reciprocity more thoroughly. Grandparents' and children's attitudes towards sex roles that specify who is primarily responsible for childcare and maintaining family bonds might be especially important in this respect. Preserving the family name might be another culturally induced motive for grandparents to bias their investments. Discriminative grandparental investments might also be explained by a mechanism of delayed reciprocity. In return for their grandparental investments, grandparents may receive more support from their children in the future (Geurts et al., 2012). A caveat is that our study did not include measures for the expected future support from specific children. Therefore, delayed reciprocity cannot be excluded as an explanation of the reported biases in grandparental investments. However, our study included measures of current support from the children to the grandparent and current support might be a good proxy for expected future support.

Recent evolutionary studies have suggested that discriminative grandparental investments might be better explained by X-chromosome relatedness than by paternity uncertainty (Fox et al., 2010, 2011; Rice et al., 2010). X-chromosome relatedness varies by the combination of the sex of the grandchild, the sex of the parent and the sex of the grandparent (Chrastil et al., 2006). Tests of the X-chromosome relatedness hypothesis have yielded positive results in pre-modern societies (Fox et al., 2010, 2011; Rice et al., 2010), but mixed results in modern societies (Chrastil et al., 2006; Rice et al., 2010; Tanskanen et al., 2011). Testing this hypothesis requires the sex of the grandchild that is cared for to be known. The sex of the grandchild that was cared for was unknown in the Chinese data. In the Dutch data the sex of the grandchild was known, but grandparents hardly varied their amount of care at the grandchild level. In $93 \%$ of the families the grandparent took care of all grandchildren of the same child equally often. This last result seems to be in line with Tanskanen et al. (2011), who analysed data from the UK and concluded that X-chromosomal relatedness does not appear to shape grandparental investments in modern societies. However, more research in modern societies with different cultural backgrounds is needed to assess the influence of Xchromosomal relatedness on grandparental investments.

A final caveat is that this study did not focus on grandparental investments in the grandchildren of stepchildren or adopted children. Research on grandparental investments in genetically unrelated grandchildren could enhance our knowledge of the importance of genetic relationships for grandparental investments. In contrast to the situation of paternity uncertainty, there is clarity about the absence of a genetic relationship between grandparents and stepchildren or adopted children. However, the Chinese sample did not include information on stepchildren and adopted children. The Dutch sample only included five adopted children. In the Dutch sample the contact of older adults with their stepchildren was extensively studied by Van der Pas \& Van Tilburg (2010). They concluded that social factors superseded genetic factors in determining the amount of contact between older parents and their biological and stepchildren. This conclusion is in line with the results of our study, which suggest 
that discriminative grandparental investments are not categorically affected by the certainty of the genetic relationship and the reproductive potential of children and, more generally, attest to the importance of testing evolutionary hypotheses in populations from diverse cultural backgrounds.

\section{References}

Almond, D. \& Edlund, L. (2007) Trivers-Willard at birth and one year: evidence from US natality data 1983-2001. Proceedings of the Royal Society B: Biological Sciences 274, 2491-2496.

Alvergne, A., Faurie, C. \& Raymond, M. (2009) Father-offspring resemblance predicts paternal investment in humans. Animal Behaviour 78, 61-69.

Anderson, K. G. (2006) How well does paternity confidence match actual paternity? Evidence from worldwide nonpaternity rates. Current Anthropology 47, 513-520.

Anderson, K. G., Kaplan, H. \& Lancaster, J. B. (2007) Confidence of paternity, divorce, and investment in children by Albuquerque men. Evolution and Human Behavior 28, 1-10.

Bishop, D. I., Meyer, B. C., Schmidt, T. M. \& Gray, B. R. (2009) Differential investment behavior between grandparents and grandchildren: the role of paternity uncertainty. Evolutionary Psychology 7, 66-77.

Boerner, K. \& Reinhardt, J. P. (2003) Giving while in need: support provided by disabled older adults. Journal of Gerontology: Social Sciences 58B, S297-304.

Boyd, R. \& Richerson, P. J. (2009) Culture and the evolution of human cooperation. Philosophical Transactions of the Royal Society B: Biological Sciences 364, 3281-3288.

Boyd, R., Richerson, P. J. \& Henrich, J. (2011) The cultural niche: why social learning is essential for human adaptation. Proceedings of the National Academy of Sciences of the USA 108, $10918-10925$.

Brown, G. R., Laland, K. N. \& Borgerhoff Mulder, M. (2009) Bateman's principles and human sex roles. Trends in Ecology \& Evolution 24, 297-304.

Buss, D. M. (1994) The Evolution of Desire: Strategies of Human Mating. Basic Books, New York.

Buss, D. M. \& Schmitt, D. P. (1993) Sexual strategies theory: an evolutionary perspective on human mating. Psychological Review 100, 204-232.

Cameron, E. Z. \& Dalerum, F. (2009) A Trivers-Willard effect in contemporary humans: malebiased sex ratios among billionaires. PLOS ONE 4, 1-4.

Cardia, E. \& Ng, S. (2003) Intergenerational time transfers and childcare. Review of Economic Dynamics 6, 431-454.

Chrastil, E. R., Getz, W. M., Euler, H. A. \& Starks, P. T. (2006) Paternity uncertainty overrides sex chromosome selection for preferential grandparenting. Evolution and Human Behavior 27, 206-223.

Coall, D. A. \& Hertwig, R. (2010) Grandparental investment: past, present, and future. Behavioral and Brain Sciences 33, 1-19.

Deeg, D. J. H., Van Tilburg, T., Smit, J. H. \& De Leeuw, E. D. (2002) Attrition in the Longitudinal Aging Study Amsterdam: the effect of differential inclusion in side studies. Journal of Clinical Epidemiology 55, 319-328.

Dimova, R. \& Wolff, F. (2011) Do downward private transfers enhance maternal labor supply? Evidence from around Europe. Journal of Population Economics 24, 911-933.

Draper, D. (2008) Bayesian multilevel analysis and MCMC. In De Leeuw, J. \& Meijer, E. (eds) Handbook of Multilevel Analysis. Springer, New York, pp. 77-139.

Dubas, J. S. (2001) How gender moderates the grandparent-grandchild relationship: a comparison of kin-keeper and kin-selector theories. Journal of Family Issues 22, 478-492. 
Eisenberg, A. R. (1988) Grandchildren's perspectives on relationships with grandparents: the influence of gender across generations. Sex Roles 19, 205-217.

Euler, H. A., Hoier, S. \& Rohde, P. A. (2001) Relationship-specific closeness of intergenerational family ties: findings from evolutionary psychology and implications for models of cultural transmission. Journal of Cross-Cultural Psychology 32, 147-158.

Euler, H. A. \& Weitzel, B. (1996) Discriminative grandparental solicitude as reproductive strategy. Human Nature 7, 39-59.

Fieder, M. \& Huber, S. (2007) The effects of sex and childlessness on the association between status and reproductive output in modern society. Evolution and Human Behavior 28, 392-398.

Fieder, M., Huber, S. \& Bookstein, F. L. (2011) Socioeconomic status, marital status and childlessness in men and women: an analysis of census data from six countries. Journal of Biosocial Science 43, 619-635.

Fingerman, K., Miller, L., Birditt, K. \& Zarit, S. (2009) Giving to the good and the needy: parental support of grown children. Journal of Marriage and Family 71, 1220-1233.

Fox, M., Johow, J. \& Knapp, L. A. (2011) The selfish grandma gene: the roles of the Xchromosome and paternity uncertainty in the evolution of grandmothering behavior and longevity. International Journal of Evolutionary Biology 2011, 1-9.

Fox, M., Sear, R., Beise, J., Ragsdale, G., Voland, E. \& Knapp, L. A. (2010) Grandma plays favourites: X-chromosome relatedness and sex-specific childhood mortality. Proceedings of the Royal Society B: Biological Sciences 277, 567-573.

Freese, J. \& Powell, B. (1999) Sociobiology, status, and parental investment in sons and daughters: testing the Trivers-Willard hypothesis. American Journal of Sociology 104, 1704-1743.

Friedman, D., Hechter, M. \& Kreager, D. (2008) A theory of the value of grandchildren. Rationality and Society 20, 31-63.

Fuller-Thomson, E. \& Minkler, M. (2001) American grandparents providing extensive child care to their grandchildren: prevalence and profile. Gerontologist 41, 201-209.

Gaulin, S. J. C. \& Robbins, C. J. (1991) Trivers-Willard effect in contemporary North American society. American Journal of Physical Anthropology 85, 61-69.

Geary, D. C. (1998) Male, Female: The Evolution of Human Sex Differences. American Psychological Association, Washington.

Geary, D. C., Vigil, J. \& Byrd-Craven, J. (2004) Evolution of human mate choice. Journal of Sex Research 41, 27-42.

Geurts, T., Poortman, A-R. \& Van Tilburg, T. G. (2012) Older parents providing child care for adult children: does it pay off? Journal of Marriage and Family 74, 239-250.

Gilding, M. (2009) Paternity uncertainty and evolutionary psychology: how a seemingly capricious occurrence fails to follow laws of greater generality. Sociology 43, 140-157.

Gray, A. (2005) The changing availability of grandparents as carers and its implications for childcare policy in the UK. Journal of Social Policy 34, 557-577.

Hank, K. \& Buber, I. (2009) Grandparents caring for their grandchildren: findings from the 2004 survey of health, ageing, and retirement in Europe. Journal of Family Issues 30, 53-73.

Hawkes, K. (2004) Human longevity: the grandmother effect. Nature 428, 128-129.

Heijkoop, M., Dubas, S. \& van Aken, M. (2009) Parent-child resemblance and kin investment: physical resemblance or personality similarity? European Journal of Developmental Psychology 6, 64-69.

Henretta, J., Hill, M., Li, W., Soldo, B. \& Wolf, D. (1997) Selection of children to provide care: the effect of earlier parental transfers. Journals of Gerontology: Psychological and Social Sciences 52, 110-119.

Hopcroft, R. L. (2005) Parental status and differential investment in sons and daughters: TriversWillard revisited. Social Forces 83, 1111-1136. 
Hopcroft, R. L. (2006) Sex, status, and reproductive success in the contemporary United States. Evolution and Human Behavior 27, 104-120.

Hoppmann, C. A. \& Klumb, P. L. (2010) Grandparental investment facilitates harmonization of work and family in employed parents: a lifespan psychological perspective. Behavioral and Brain Sciences 33, 27-28.

Hox, J. J. (2002) Multilevel Analysis: Techniques and Applications. Lawrence Erlbaum, Mahwah.

Hrdy, S. B. (2005) Cooperative breeders with an ace in the hole. In Voland, E., Chasiotis, A. \& Schiefenhövel, W. (eds) Grandmotherhood: The Evolutionary Significance of the Second Half of Female Life. Rutgers University Press, New Brunswick, pp. 295-317.

Johnson, J. O. (2005) Who's Minding the Kids? Child Care Arrangements: Winter 2002. US Census Bureau, Washington.

Kaptijn, R. \& Thomese, F. (2010) Fitness effects of grandparental investments in contemporary low-risk societies. Behavioral and Brain Sciences 33, 29-30.

Kaptijn, R., Thomese, F., Van Tilburg, T. \& Liefbroer, A. (2010) How grandparents matter: support for the cooperative breeding hypothesis in a contemporary Dutch population. Human Nature 21, 393-405.

Keller, M. C., Nesse, R. M. \& Hofferth, S. (2001) The Trivers-Willard hypothesis of parental investment: no effect in the contemporary United States. Evolution and Human Behavior 22, $343-360$.

King, V. \& Elder, G. H. Jr (1995) American children view their grandparents: linked lives across three rural generations. Journal of Marriage and the Family 57, 165-178.

King, V., Silverstein, M., Elder, G. H. Jr, Bengtson, V. L. \& Conger, R. D. (2003) Relations with grandparents: rural midwest versus urban Southern California. Journal of Family Issues 24, 1044-1069.

Knipscheer, C. P. M., De Jong Gierveld, J., Van Tilburg, T. \& Dykstra, P. A. (eds) (1995) Living Arrangements and Social Networks of Older Adults. VU University Press, Amsterdam.

Kohli, M. \& Künemund, H. (2003) Intergenerational transfers in the family: what motivates giving? In Bengtson, V. L. \& Lowenstein, A. (eds) Global Aging and Challenges to Families. Aldine de Gruyter, New York, pp. 123-142.

Koziel, S. \& Ulijaszek, S. J. (2001) Waiting for Trivers and Willard: do the rich really favor sons? American Journal of Physical Anthropology 115, 71-79.

Kunemund, H. \& Rein, M. (1999) There is more to receiving than needing: theoretical arguments and empirical explorations of crowding in and crowding out. Ageing and Society 19, 93-121.

Laham, S. M., Gonsalkorale, K. \& Von Hippel, W. (2005) Darwinian grandparenting: preferential investment in more certain kin. Personality and Social Psychology Bulletin 31, 63-72.

Lahdenpera, M., Lummaa, V., Helle, S., Tremblay, M. \& Russell, A. F. (2004) Fitness benefits of prolonged post-reproductive lifespan in women. Nature 428, 178-181.

Lahdenpera, M., Russell, A. F. \& Lummaa, V. (2007) Selection for long lifespan in men: benefits of grandfathering? Proceedings of the Royal Society B: Biological Sciences 274, 2437-2444.

Lai-wan, C. C., Blyth, E. \& Hoi-yan, C. C. (2006) Attitudes to and practices regarding sex selection in China. Prenatal Diagnosis 26, 610-613.

Liao, T. F. (1994) Interpreting Probability Models: Logit, Probit, and Other Generalized Linear Models. Sage, Thousand Oaks.

McGarry, K. \& Schoeni, R. (1997) Transfer behavior within the family: results from the asset and health dynamics study. Journals of Gerontology: Psychological and Social Sciences 52, 82-92.

Magnuson, A., Bodin, L. \& Montgomery, S. M. (2007) Father's occupation and sex ratio of offspring. Scandinavian Journal of Public Health 35, 454-459.

Michalski, R. L. \& Shackelford, T. K. (2005) Grandparental investment as a function of relational uncertainty and emotional closeness with parents. Human Nature 16, 293-305. 
Nettle, D. \& Pollet, T. V. (2008) Natural selection on male wealth in humans. The American Naturalist 172, 658-666.

Nowak, M. A. (2006) Five rules for the evolution of cooperation. Science 314, 1560-1563.

Pashos, A. (2000) Does paternal uncertainty explain discriminative grandparental solicitude? A cross-cultural study in Greece and Germany. Evolution and Human Behavior 21, 97-109.

Pillemer, K. \& Suitor, J. J. (2006) Making choices: a within-family study of caregiver selection. The Gerontologist 46, 439-448.

Pollet, T. V., Nelissen, M. \& Nettle, D. (2009) Lineage based differences in grandparental investment: evidence from a large British cohort study. Journal of Biosocial Science 41, 355-379.

Pollet, T. V., Nettle, D. \& Nelissen, M. (2006) Contact frequencies between grandparents and grandchildren in a modern society: estimates of the impact of paternity uncertainty. Journal of Cultural and Evolutionary Psychology 4, 203-213.

Pollet, T. V., Nettle, D. \& Nelissen, M. (2007) Maternal grandmothers do go the extra mile: factoring distance and lineage into differential contact with children. Evolutionary Psychology 5, 832-843.

Portegijs, W., Clö̈n, M., Ooms, I. \& Eggink, E. (2006) Hoe het werkt met kinderen: Moeders over kinderopvang en werk. Sociaal en Cultureel Planbureau, Den Haag.

Rasbash, J., Steele, F., Browne, W. \& Prosser, B. (2004) A User's Guide to MLwiN. Centre for Multilevel Modelling, University of Bristol, Bristol.

Rice, W. R., Gavrilets, S. \& Friberg, U. (2010) The evolution of sex-specific grandparental harm. Proceedings of the Royal Society B: Biological Sciences 277, 2727-2735.

Richerson, P. J. \& Boyd, R. (2004) Not by Genes Alone: How Culture Transformed Human Evolution. University of Chicago Press, Chicago.

Salmon, C. A. (1999) On the impact of sex and birth order on contact with kin. Human Nature 10, 183-197.

Sear, R. \& Mace, R. (2008) Who keeps children alive? A review of the effects of kin on child survival. Evolution and Human Behavior 29, 1-18.

Silverstein, M. (2007) Do bioevolutionary forces shape intergenerational transfers? Detecting evidence in contemporary survey data. In Booth, A. (ed.) Caring and Exchange Within and Across Generations. Urban Institute Press, Washington, pp. 127-144.

Silverstein, M., Conroy, S. J., Wang, H. T., Giarrusso, R. \& Bengtson, V. L. (2002) Reciprocity in parent-child relations over the adult life course. Journal of Gerontology: Social Sciences 57B, S3-13.

Strassmann, B. \& Garrard, W. (2011) Alternatives to the grandmother hypothesis. Human Nature 22, 201-222.

Suitor, J. J. \& Pillemer, K. (2000) Did mom really love you best? Developmental histories, status transitions, and parental favoritism in later life families. Motivation and Emotion 24, 105-120.

Suitor, J. J., Pillemer, K. \& Sechrist, J. (2006) Within-family differences in mothers' support to adult children. Journal of Gerontology: Social Sciences 61B, S10-17.

Suitor, J. J., Sechrist, J. \& Pillemer, K. (2007) Within-family differences in mothers' support to adult children in black and white families. Research on Aging 29, 410-435.

Tanskanen, A. O., Rotkirch, A. \& Danielsbacka, M. (2011) Do grandparents favor granddaughters? Biased grandparental investment in UK. Evolution and Human Behavior 32, 407-415.

Tooby, J. \& Cosmides, L. (1990) The past explains the present: emotional adaptations and the structure of ancestral environments. Ethology and Sociobiology 11, 375-424.

Tooby, J. \& Cosmides, L. (1992) The psychological foundations of culture. In Barkow, J., Tooby, J. \& Cosmides, L. (eds) The Adapted Mind: Evolutionary Psychology and the Generation of Culture. Oxford University Press, New York, pp. 19-136.

Trivers, R. L. (1971) The evolution of reciprocal altruism. The Quarterly Review of Biology 46, $35-57$. 
Trivers, R. L. (1972) Parental investment and sexual selection. In Campbell, B. (ed.) Sexual Selection and the Descent of Man (1871-1971). Aldline Publishing Company, Chicago, pp. 136-179.

Trivers, R. L. \& Willard, D. E. (1973) Natural-selection of parental ability to vary sex-ratio of offspring. Science 179, 90-92.

Tuljapurkar, S. D., Puleston, C. O. \& Gurven, M. D. (2007) Why men matter: mating patterns drive evolution of human lifespan. PloS ONE 2, 1-6.

Uhlenberg, P. (1996) Mortality decline in the twentieth century and supply of kin over the life course. The Gerontologist 36, 681.

Van der Pas, S. \& Van Tilburg, T. (2010) The influence of family structure on the contact between older parents and their adult biological children and stepchildren in the Netherlands. Journal of Gerontology: Social Sciences 65B, 236-245.

Waynforth, D. (2012) Grandparental investment and reproductive decisions in the longitudinal 1970 British cohort study. Proceedings of the Royal Society B: Biological Sciences 279, 11551160.

Wheelock, J. \& Jones, K. (2002) 'Grandparents are the next best thing': informal childcare for working parents in urban Britain. Journal of Social Policy 31, 441-463. 